\title{
PROPOSTA DE INDICADORES DE AVALIAÇÃO DO PPSUS E DE OUTROS PROGRAMAS DE FOMENTO DESCENTRALIZADO SOB GESTÃO DO CNPQ
}

\section{Proposal for PPSUS Evaluation Indicators and other Decentralized Programs Managed by CNPq}

\author{
Suzana Rachel de Oliveira* \\ Carolina Bittencourt Gomes** \\ Luciana Calabró***
}

\begin{abstract}
Resumo: A descentralização das políticas de Ciência, Tecnologia e Inovação tem sido uma estratégia adotada pelo Conselho Nacional de Desenvolvimento Científico e Tecnológico para redução das desigualdades regionais nessa área. Visando a redução das desigualdades na área específica de Ciência, Tecnologia e Inovação em saúde, o Ministério da Saúde criou, em 2004, o Programa Pesquisa para o SUS: gestão compartilhada em saúde. Para operacionalizar o Programa, o $\mathrm{CNPq}$ firma convênios com as Fundações de Amparo à Pesquisa nos estados. Assim como o PPSUS, há outros programas sob gestão do CNPq em que é utilizado também este mesmo instrumento com as FAPs. A ausência de indicadores de insumo e resultado para acompanhamento e avaliação das ações de fomento descentralizado suscitou a realização deste estudo cujo objetivo foi elaborar indicadores de insumo e resultado para o PPSUS e para outros programas de fomento descentralizado, baseados na técnica Conferência de Consenso, descrevendo o caminho percorrido nessa elaboração. Foi realizada uma pesquisa metodológica, de abordagem quali-quantitativa. A Conferência de Consenso propiciou, como resultado, a obtenção de duas Matrizes: uma contendo indicadores de insumo e resultado para o PPSUS e outra para os demais programas. Concluiu-se que apesar da dificuldade na tentativa de se obter indicadores comuns para as ações operacionalizadas por meio do fomento descentralizado, tendo em vista a diversidade de objetivos e arranjos específicos de cada programa, foi possível propor um rol de indicadores aos gestores do CNPq.
\end{abstract}

Palavras-chave: Indicadores de Ciência, Tecnologia e Inovação. PPSUS. Conferência de Consenso.

Abstract: The decentralization of Science, Technology and Innovation policies has been a strategy adopted by the National Council for Scientific and Technological Development to

\footnotetext{
* Doutoranda do Programa de Educação em Ciências da Universidade Federal do Rio Grande do Sul (UFRGS), Analista em Ciência e Tecnologia do Conselho Nacional de Desenvolvimento Científico e Tecnológico (CNPq), Orcid: https://orcid.org/0000-0002-3275-9199, e-mail: suzanarachelo@gmail.com.

** Mestranda do Programa de Educação em Ciências da UFRGS, Especialista em Vigilância Sanitária, Atenção Farmacêutica e Farmacologia, Analista em Ciência e Tecnologia do CNPq, Orcid: https://orcid.org/0000-00032928-4994, e-mail: carol.gomes@gmail.com.

*** Doutora em Educação em Ciências pela UFRGS, Orcid: https://orcid.org/0000-0001-6669-1789, e-mail: luciana.calabro@ufrgs.br.
} 
reduce regional inequalities in this area. In order to reduce inequalities in the specific field of Science, Technology and Innovation in health, the Ministry of Health created, in 2004, the Research Program for SUS: shared management in health. To operationalize the Program, the National Council for Scientific and Technological Development signs agreements with the Foundations of Support to Research in the STATES. As well as PPSUS, there are other programs under the management of $\mathrm{CNPq}$ in which it also uses this same instrument with the FAPs. This study had been done due to the lack of input and output indicators for monitoring and evaluating $\mathrm{CNPq}^{\prime}$ 's decentralized funding actions. The objective of the study is to develop input and output indicators for PPSUS and other decentralized funding programs based on the Consensus Conference technique and the pathway followed in this elaboration was described. It was a methodological research with both quantitative and qualitative approaches. As a result, this technique made it possible to obtain two Matrices: one containing input and output indicators for PPSUS and the other one for other programs. Although we can emphasize the difficulty in trying to obtain common indicators due to the diversity of objectives and specific arrangements of each program, it was possible to offer some indicators for $\mathrm{CNPq}^{\prime}$ s managers.

Keywords: Science, Technology and Innovation indicators. PPSUS. Consensus Conference.

\section{Introdução}

Ciência e Tecnologia são componentes básicos no planejamento nacional em busca de desenvolvimento econômico, preservação do meio ambiente e diminuição das desigualdades sociais, bem como são soluções para os diversos problemas na sociedade, como emprego, educação, habitação, saneamento, saúde e outros que dependem, portanto, de pesquisa (SILVA, 2000). No entanto, políticas de Ciência, Tecnologia e Inovação (C, T\&I) estritamente centralizadas são pouco eficientes para alcançar o objetivo de reverter para a sociedade os resultados oriundos dos investimentos no desenvolvimento científico, considerando a diversidade econômica e social no território nacional. Portanto, faz-se necessário que as políticas de $\mathrm{C}$, T\&I sejam descentralizadas, mas sem seguir um formato único, visto que são direcionadas a realidades locais díspares e, para serem bem sucedidas, precisam contar com a adesão dos atores locais e se adaptar às diferentes realidades (BALBACHEVSKY, 2008).

A descentralização das políticas de apoio à Ciência, Tecnologia e Inovação tem assumido um papel crucial no país, considerando a crescente importância da inovação tecnológica na agenda de desenvolvimento econômico e social, bem como o reconhecimento de que parte importante dessa inovação possui caráter local e de que é necessária a disponibilização de recursos humanos qualificados para promovê-la (CGEE, 2008). Segundo Silva (2000), os países desenvolvidos tendem cada vez mais a descentralizar a sua capacidade em produzir conhecimentos e recursos humanos, criando, assim, condições para que todas as regiões se beneficiem com o recebimento de recursos para as ações de Ciência, Tecnologia e Inovação. A falta de políticas que apoiem a descentralização pode agravar a desigualdade entre as regiões brasileiras, dada a dificuldade das regiões menos favorecidas, como Norte, Nordeste e Centro-Oeste, em competir pelos recursos federais e em formar e fixar novos cientistas.

A demonstração das desigualdades entre as regiões brasileiras na área de pesquisa em C, T\&I no Brasil pode ser exemplificada na distribuição dos grupos de pesquisa nacionais. Segundo dados do Diretório do Grupo de Pesquisas do CNPq, conforme censo de 2016, o número de Doutores na região Sudeste era de 67.514 (46\% do total) e na Região Sul era de 31.318 (21,3\% do total). Juntas, as regiões Sudeste e Sul possuíam, então, 67,3\% do total de 
pesquisadores Doutores cadastrados no Diretório, enquanto às demais regiões (Norte, Nordeste e Centro-Oeste) restavam 32,7\%.

A redução das desigualdades regionais na produção e no acesso à $C$, $T \& I$ constitui, inclusive, um desafio a ser superado pela Estratégia Nacional de Ciência, Tecnologia e Inovação 2016/2022, que é documento estratégico para o planejamento do Sistema Nacional de Ciência, Tecnologia e Inovação (BRASIL, 2018), mostrando a preocupação constante do Ministério da Ciência, Tecnologia e Inovações com o tema desigualdade regional.

No caso específico de redução das desigualdades regionais na área de Ciência, Tecnologia e Inovação em saúde, o Ministério da Saúde criou, em 2004, o Programa Pesquisa para o SUS: gestão compartilhada em saúde (PPSUS). O PPSUS é operacionalizado de forma descentralizada e conta com a parceria do Conselho Nacional de Desenvolvimento Científico Tecnológico (CNPq), das Fundações de Amparo à Pesquisa (FAP) e das Secretarias Estaduais de Saúde (SES).

Contudo, o processo de descentralização de qualquer política pública é complexo, pois se refere à distribuição de funções - responsabilidade pela execução e/ou autoridade decisória - entre níveis de governo (federal, estadual e municipal) e entre esses níveis e instituições do setor privado (CGEE, 2010), além das elevadas desigualdades regionais, que são um desafio adicional.

A responsabilidade pela execução do PPSUS é da FAP, em consonância com as Diretrizes Técnicas do Programa (BRASIL, 2014). Já em relação à autoridade decisória, o PPSUS propõe uma gestão compartilhada entre Ministério da Saúde, CNPq, FAP e SES em que cada parceiro possui suas responsabilidades definidas nas Diretrizes e não há uma hierarquia.

Em 2019, o Ministério da Saúde instituiu oficialmente o PPSUS por meio da Portaria 3.020 , de 19 de novembro de 2019, que estabeleceu os seguintes objetivos para o Programa: I - apoiar financeiramente o desenvolvimento de pesquisas científicas, tecnológicas e/ou de inovação que objetivem contribuir para a resolução de problemas de saúde da população dos Estados e do Distrito Federal e para o fortalecimento da gestão do SUS; II - promover a aproximação dos sistemas de saúde com os de ciência e tecnologia locais; e III - reduzir as desigualdades regionais no campo da ciência, tecnologia e inovação em saúde no país (BRASIL, 2019).

O PPSUS é uma política que vem sendo fortalecida e continuada pelo Ministério da Saúde. O Programa conta com 07 Edições (2004; 2006; 2008; 2011; 2012; 2015-2016-2017 e 2020), sendo que a última foi iniciada em julho/2020. O CNPq operacionaliza outros programas de fomento descentralizado em parcerias com as FAPs, como o Programa de Apoio a Núcleos de Excelência (PRONEX). Tais programas possuem, em comum, o fato de serem operacionalizados mediante firmatura de convênios.

Apesar de as parcerias do CNPQ com as FAPs não serem recentes, não foram localizados indicadores para monitoramento e avaliação das ações de fomento descentralizado executadas no CNPq, o que suscitou a realização deste estudo. Partiu-se do pressuposto de que os programas poderiam ter alguma forma de monitoramento comum, visto que se utilizam do mesmo meio de operacionalização: via convênios.

A Portaria Interministerial $n^{\circ} 424 / 2016$, de 30 de dezembro de 2016, estabelece que convênio é o instrumento que disciplina a transferência de recursos financeiros de órgãos ou entidades da Administração Pública Federal, direta ou indireta, para órgãos ou entidades da Administração Pública Estadual, Distrital ou Municipal, direta ou indireta, consórcios públicos, 
ou ainda, entidades privadas sem fins lucrativos, visando à execução de projeto ou atividade de interesse recíproco, em regime de mútua cooperação (BRASIL, 2016).

O Quadro 01 relaciona os principais programas de fomento descentralizado operacionalizados no $\mathrm{CNPq}$, por meio de convênios com as FAPs, sob responsabilidade das duas áreas técnicas envolvidas: Coordenação-Geral do Programa de Pesquisa em Saúde e Coordenação de Parcerias Estaduais. Os programas relacionados são os que possuem maior número de convênios ativos.

Quadro 1 - Principais Programas de Fomento Descentralizado operacionalizados no CNPq mediante convênios com as Fundações de Amparo à Pesquisa

\begin{tabular}{|c|c|c|}
\hline Programa & $\begin{array}{l}\text { Objetivos, temas prioritários e } \\
\text { especificidades operacionais }\end{array}$ & Atores relevantes \\
\hline $\begin{array}{l}\text { Programa de Infraestrutura para } \\
\text { Jovens Pesquisadores ou Programa } \\
\text { Primeiros Projetos - PPP }\end{array}$ & $\begin{array}{l}\text { Apoiar pesquisadores com, no } \\
\text { máximo, } 8 \text { (oito) anos de } \\
\text { doutoramento e que não sejam } \\
\text { bolsistas de produtividade do CNPq, } \\
\text { por meio do fomento a projetos de P, } \\
\text { D\&I que preconizem a aquisição, } \\
\text { instalação, modernização ou } \\
\text { recuperação da infraestrutura de } \\
\text { pesquisa científica e tecnológica nas } \\
\text { Instituições Científicas, } \\
\text { Tecnológicas e de Inovação (ICTs), } \\
\text { conforme definidas pela lei no } \\
\text { 10.973/2004 e suas alterações. }\end{array}$ & CNPq, FAPs e ICTs \\
\hline $\begin{array}{l}\text { Programa de Apoio a Núcleos de } \\
\text { Excelência - PRONEX }\end{array}$ & $\begin{array}{l}\text { Criado em 1996, o PRONEX é um } \\
\text { instrumento de estímulo à pesquisa e } \\
\text { ao desenvolvimento científico e } \\
\text { tecnológico do país, por meio de } \\
\text { apoio continuado e adicional aos } \\
\text { demais instrumentos disponíveis, a } \\
\text { grupos de alta competência, com } \\
\text { liderança e papel nucleador na área } \\
\text { de sua atuação. Núcleos de } \\
\text { Excelência são grupos organizados } \\
\text { de pesquisadores e técnicos de alto } \\
\text { nível, em permanente interação, com } \\
\text { reconhecida competência e tradição } \\
\text { em suas áreas de atuação técnico- } \\
\text { científica, capazes de funcionar } \\
\text { como fonte geradora e } \\
\text { transformadora de conhecimento } \\
\text { científico-tecnológico para aplicação } \\
\text { em programas e projetos de } \\
\text { relevância ao desenvolvimento do } \\
\text { país. A partir de 2003, o PRONEX } \\
\text { passou a ser executado em parceria } \\
\text { com as FAPs. }\end{array}$ & CNPq, FAPs, ICTs \\
\hline $\begin{array}{l}\text { Programa de Apoio a Núcleos } \\
\text { Emergentes de Pesquisa - } \\
\text { PRONEM }\end{array}$ & $\begin{array}{l}\text { Apoiar pesquisadores com, no } \\
\text { mínimo, } 3 \text { (três) e, no máximo, } 8 \\
\text { (oito) anos de doutoramento, por } \\
\text { intermédio do fomento a projetos de } \\
\text { P, D\&I que preconizem a formação } \\
\text { de núcleos de pesquisa, envolvendo } \\
\text { intercâmbio de pesquisadores, } \\
\text { pesquisa }\end{array}$ & CNPq, FAPs, ICTs \\
\hline
\end{tabular}




\begin{tabular}{|c|c|c|}
\hline & $\begin{array}{l}\text { transferência de conhecimento e } \\
\text { melhoria da infraestrutura de } \\
\text { pesquisa científica e tecnológica nas } \\
\text { Instituições Científicas, } \\
\text { Tecnológicas e de Inovação (ICTs), } \\
\text { conforme definidas pela Lei n } \\
\text { 10.973/2004 e suas alterações. }\end{array}$ & \\
\hline $\begin{array}{l}\text { Programa Pesquisa para o SUS: } \\
\text { gestão compartilhada em saúde - } \\
\text { PPSUS }\end{array}$ & $\begin{array}{l}\text { O Ministério da Saúde, por } \\
\text { intermédio do Decit/SCTIE, } \\
\text { dissemina o fomento descentralizado } \\
\text { à pesquisa nos estados, por meio do } \\
\text { PPSUS. Para isso, são repassados, } \\
\text { via CNPq, recursos financeiros às } \\
\text { Fundações de Amparo à Pesquisa } \\
\text { (FAPs) que, em parceria com as } \\
\text { respectivas secretarias estaduais de } \\
\text { saúde (SES), publicam editais para } \\
\text { seleção de projetos relevantes para o } \\
\text { Sistema Único de Saúde (SUS). }\end{array}$ & CNPq, FAPs, SES \\
\hline
\end{tabular}

Fonte: Elaborado pelas autoras (2021).

Segundo Ohayon e Rosenberg (2014), a construção de indicadores em C, T\&I é justificada pela necessidade de melhor gerenciamento dos recursos escassos relacionados à área e pela importância no auxílio aos gestores nas avaliações das atividades de Ciência, Tecnologia e Inovação.

Considerando a importância de se avaliar essa forma de fomento existente no CNPq, o presente estudo pretendeu elaborar indicadores para avaliar o PPSUS, a partir da proposta da técnica de Conferência de Consenso, e descrever o caminho percorrido nessa elaboração. Os indicadores poderão orientar as ações do CNPq na gestão do PPSUS e de outros programas de fomento descentralizado operacionalizados via convênios com as FAPs.

\section{Metodologia}

Trata-se de uma pesquisa metodológica, de abordagem quali-quantitativa. Este tipo de estudo refere-se a instrumentos de captação, coleta de dados e/ou intervenção na realidade. Estão envolvidos os caminhos, formas, maneiras e procedimentos para atender a um determinado fim (TOBAR; YALOUR, 2001). No presente caso, o instrumento a ser construído é uma Matriz de Indicadores que possa ser utilizada na avaliação do PPSUS e, se adaptada, para outros programas de fomento descentralizado.

Foi utilizada a técnica Conferência de Consenso, a qual concilia uma discussão ampla e aberta entre especialistas no sentido de obter um consenso, utilizada especialmente no campo da saúde para definição de indicadores para avaliação de programas (SOUZA; VIEIRA-DASILVA; HARTZ, 2005). Além disso, a Conferência de Consenso, proposta por Souza, Vieirada-Silva e Hartz (2005), consegue conciliar a possibilidade de discussão aberta entre os especialistas e a preservação do anonimato dos participantes, dentro de uma logística viável.

Foi realizada a seleção, por meio de uma amostra de conveniência, de um grupo de cinco especialistas, para se promoverem as discussões em busca de consenso. Não foi selecionado um grupo extenso, para que fosse assegurada a participação individual numa ampla e aprofundada discussão sobre os indicadores que mais se adequassem às necessidades da gestão do PPSUS e de outros programas de fomento descentralizado no âmbito do CNPq. Por isso, 
todos os especialistas selecionados são servidores efetivos do $\mathrm{CNPq}$, com experiência superior de 10 anos na gestão pública e possuem expertise envolvendo os temas: gestão do PPSUS, gestão de demais programas por meio de parceria estaduais e gestão na área de estatística e indicadores.

Os indicadores propostos foram divididos em indicadores de insumo (input) e de resultado (output). Segundo Cavalcante e Aquino (2008), os indicadores de insumo estão relacionados aos recursos humanos, físicos e financeiros alocados nas atividades científica e tecnológica. Os indicadores de resultado procuram aferir as repercussões dessas atividades sobre os indicadores econômicos e sociais de um país ou uma região. Segundo os autores, os indicadores de resultado são escassos e de difíceis aferições e, por isso, são utilizadas, em geral, medidas indiretas de resultados das atividades de C\&T, como publicações científicas e registros de patentes.

O processo de elaboração da Matriz e realização da Conferência de Consenso foi desenvolvido em quatro etapas, descritas a seguir:

\subsection{Primeira etapa}

Foi realizada uma análise documental para elaboração de uma Matriz inicial que contemplasse indicadores de insumo (input) e resultado (output). Não foram localizados documentos oficiais estabelecendo indicadores específicos para o PPSUS nem para demais programas de fomento descentralizado.

Os indicadores propostos foram adaptados a partir dos estudos de: Oliveira (2008), que sugeriu uma matriz avaliativa para avaliação do PPSUS, porém não restrita às ações desempenhadas pelo CNPq; Francisco (2002), que sugeriu indicadores para avaliação de projetos de pesquisa contratados por Fundações de Amparo à Pesquisa; e Melo, Santana e Silva (2018), que sugeriram indicadores para análise da eficiência da infraestrutura de pesquisa das regiões brasileiras (indicadores de insumo) em transformar-se em indicadores de resultado. $\mathrm{O}$ intuito era fornecer ao CNPq um instrumento de fácil mensuração e utilização pelos gestores.

\subsection{Segunda etapa}

A Matriz inicial foi enviada, via correio eletrônico, para os cinco especialistas. Foi-lhes solicitada a avaliação quanto à importância dos indicadores propostos, com atribuição de notas de 0 a 10, sendo que 10 correspondia à importância máxima e 0 significava a exclusão do indicador. Havia a possibilidade de os especialistas registrarem suas observações, comentários ou até mesmo a inclusão/substituição de indicadores. O prazo para devolução da Matriz pontuada foi de 15 dias. Nesta etapa, foi preservado o anonimato dos respondentes.

Após recebimento de todas as avaliações, as notas dos especialistas foram consolidadas em uma nova Matriz, contendo as sugestões de readequações. As notas foram consolidadas no programa Microsoft Excel e, para cada indicador, calculou-se a média aritmética (medida da sua importância) e o desvio-padrão (estimativa do grau de consenso entre os especialistas). Nesse momento, não se excluiu nenhum indicador para que todas as informações pudessem ser discutidas durante a etapa da realização da conferência.

\subsection{Terceira etapa}


A conferência de consenso foi realizada em 23/02/2021, via videoconferência, em razão da pandemia de COVID-19. Todos os cinco especialistas compareceram ao evento e neste momento foi apresentada a Matriz contendo as diversas sugestões/os diversos comentários sugeridos. Esta etapa propiciou uma rica discussão entre os especialistas, os quais puderam expressar suas dúvidas, sugestões e expectativas sobre os indicadores. Os principais pontos discutidos serão detalhados na seção Resultados e Discussão.

Após realização da conferência, a Matriz foi reorganizada e reenviada aos especialistas para nova rodada de avaliação individual com atribuição de notas, seguindo a mesma lógica de pontuação descrita na Primeira Etapa. O anonimato dos respondentes foi preservado.

\subsection{Quarta Etapa}

Por fim, após o recebimento das respostas sobre a realização da conferência, foram novamente calculados a média aritmética e o desvio-padrão para todos os indicadores, utilizando-se o programa Microsoft Excel. Os valores adotados para a seleção dos indicadores, para compor a Matriz final, foram de acordo com a metodologia proposta por Souza, Vieirada-Silva e Hartz (2005) descrita abaixo:

a) Todo indicador com média igual ou superior a sete foi considerado como importante. Abaixo disso, seria pouco importante, não devendo fazer parte da Matriz.

b) Todo indicador com desvio-padrão inferior a três foi considerado consensual. Caso fosse também importante, o critério/indicador deveria ser incluído na Matriz.

c) Os indicadores com média igual ou superior a sete e desvio-padrão igual ou superior a três, apesar de importantes, não deveriam compor a Matriz, pois não seriam consensuais.

Ainda segundo os autores, quanto maior a média aritmética, maior a importância do critério, e o desvio-padrão permite a estimativa do grau de consenso.

\section{Resultados e Discussão}

É importante descrever pontos relevantes discutidos entre os especialistas na reunião ocorrida de forma remota, como, a recomendação que as áreas gestoras poderão estabelecer resultados específicos considerando os objetivos de cada programa. Além disso, alguns indicadores já foram definidos, em consenso, que podiam ser retirados ou adaptados da Matriz inicial. Manteve-se o objetivo de fornecer indicadores úteis para a gestão do CNPq e que dependessem, o mínimo possível, de informações enviadas pelas FAPs.

Os especialistas recomendaram a retirada dos indicadores relacionados à formação de recursos humanos, sejam eles de insumo ou resultado, pois este não seria o objetivo específico do PPSUS nem de outros programas de fomento descentralizado. O CNPq possui, por exemplo, chamada exclusiva para formação de Mestres e Doutores, portanto tais indicadores deveriam ser usados para essas ações voltadas para tal objetivo, pois os números poderiam fornecer informações mais confiáveis para análises comparativas. Ressalta-se que o PPSUS, em todas as suas edições, nunca financiou bolsas com o recurso repassado pela União. Contudo, o financiamento de bolsas é opcional para os estados e muitos deles não as financiam. O mesmo se aplica aos demais programas.

Foi sugerido, em comum acordo, a retirada de indicadores de insumo relacionados ao número de pesquisadores/estudantes envolvidos nos projetos, pois esta informação não é 
padronizada pelas FAPs no momento de submissão dos projetos, sejam do PPSUS ou de outros programas. Alertou-se também que nem toda FAP teria condições de saber se o pesquisador contemplado estaria recebendo recursos pela primeira vez, sendo inviável a permanência do indicador proposto para verificação do número de novos pesquisadores contemplados. O CNPq também não teria como obter esta informação, já que só tem acesso ao que foi concedido por meio de seus auxílios e bolsas próprios.

Em relação aos indicadores de resultado do PPSUS, foi definido que o $\mathrm{CNPq}$ não buscaria a almejada incorporação ao SUS, pois há a compreensão de que são muitos os fatores implicados. Souza e Contandriopoulos (2004) destacam alguns desses obstáculos para uso do conhecimento científico na tomada de decisão pelos gestores da área da saúde: muitos estudos apresentam resultados inconclusivos; a aplicabilidade dos resultados de muitas pesquisas não é facilmente perceptível; as pesquisas são feitas por meio de projetos específicos e isolados e às vezes, os resultados de duas pesquisas válidas são contraditórios entre si; a tomada de decisão pelos gestores não é um processo exclusivamente racional e toda decisão é contextualizada; a aprendizagem dos novos conhecimentos é um processo complexo que não depende somente do acesso à informação e, às vezes, não levar em consideração uma informação disponível é a opção politicamente mais adequada.

Diante disso, focou-se nos resultados relacionados à produção científica, igualmente relevante para os outros programas. Contudo, convém lembrar o problema de temporalidade quanto a esta questão, considerando que a publicação dos resultados de pesquisas pode ocorrer até cinco anos após o término do projeto (NICKEL et al., 2017). O atraso existente entre o financiamento da pesquisa e os resultados obtidos também havia sido reportado por Banzi et al. (2011), assim como a falta de padronização na definição do que seriam tais resultados, em se tratando das pesquisas na área da saúde. Outro aspecto relatado pelos especialistas foi o fato de que o pesquisador não informaria no Lattes a produção científica relacionada a determinado financiamento recebido; o mesmo projeto poderia ter recebido múltiplas fontes de financiamento, não teria como estabelecer uma relação direta com determinado programa.

Para minimizar esse problema, sugeriu-se que se trabalhasse, então, com a média da produção científica nos cinco anos anteriores e cinco anos posteriores ao ano de contemplação pelo PPSUS e demais programas e não com o número absoluto. Desse modo, poder-se-ia ter uma noção do impacto do financiamento recebido pelo pesquisador em sua produção científica. $\mathrm{Na}$ visão dos especialistas, visualizar os efeitos nas médias no intervalo proposto, poderia ser um dado mais interessante que o número absoluto de artigos completos publicados e incluiria a produção decorrente da pesquisa, ocorrida mesmo após sua conclusão.

Convencionou-se que, cada área gestora no $\mathrm{CNPq}$, definiria qual tipo de produção científica seria escolhida por programa, sendo que artigos científicos completos publicados foi mantido para o PPSUS e demais programas. Da mesma forma, em relação às patentes, cada área julgaria se os indicadores relacionados a patentes solicitadas/concedidas seriam pertinentes ao programa a ser analisado e sugeriu-se a retirada. Com a retirada dos indicadores baseados no número absoluto de artigos científicos e patentes, tornou-se inviável o uso dos indicadores de produtividade, entre as regiões, adaptados do estudo desenvolvido por Melo, Santana e Silva (2018).

Ainda sobre os indicadores de resultado, sugeriu-se a inclusão dos seguintes indicadores em relação ao número de projetos financiados, tanto para o PPSUS como para outros programas: demanda bruta de projetos submetidos, por UF e número de projetos financiados, a cada R \$ 100.000,00 investidos, por UF. Dessa maneira, poderia se ter noção em que medida os programas contribuíam no atendimento da demanda submetida e poder-se-ia verificar não 
somente o valor absoluto do número de projetos financiados, mas também quanto representa este valor, por UF, considerando determinado valor investido.

A Tabela 1 mostra a Matriz final, contendo os indicadores de insumo (input) e resultado (output) para o PPSUS, descrição, fonte de obtenção dos dados, média e desvio-padrão obtidos após a quarta etapa. A Tabela 2 mostra os mesmos dados, porém que podem ser utilizados para demais programas.

Tabela 1 - Matriz final com indicadores de insumo e resultado propostos para avaliação do PPSUS

\begin{tabular}{|c|c|c|c|c|c|}
\hline $\begin{array}{c}\text { Tipo de } \\
\text { Indicador }\end{array}$ & Indicador & Descrição & $\begin{array}{c}\text { Fonte de } \\
\text { Informação }\end{array}$ & Média & $\begin{array}{l}\text { Desvio- } \\
\text { Padrão }\end{array}$ \\
\hline \multirow{7}{*}{$\begin{array}{l}\text { Insumo } \\
\text { (input) }\end{array}$} & $\begin{array}{l}\text { Total de recursos financeiros federais } \\
\text { por UF, por Edição do PPSUS }\end{array}$ & $\begin{array}{c}\text { Valor de recursos federais } \\
\text { transferidos à UF em cada Edição } \\
\text { do PPSUS }\end{array}$ & $\mathrm{CNPq}$ & 8,00 & 2,12 \\
\hline & $\begin{array}{l}\text { Total de recursos financeiros } \\
\text { estaduais por UF, por Edição do } \\
\text { PPSUS }\end{array}$ & $\begin{array}{c}\text { Valor de recursos estaduais } \\
\text { transferidos por UF em cada Edição } \\
\text { do PPSUS }\end{array}$ & $\mathrm{CNPq}$ & 8,00 & 2,12 \\
\hline & $\begin{array}{l}\text { Número de instituições participantes } \\
\text { por UF, por Edição do PPSUS }\end{array}$ & $\begin{array}{c}\text { Número de instituições } \\
\text { participantes nos projetos } \\
\text { financiados, por UF, por Edição do } \\
\text { PPSUS }\end{array}$ & $\mathrm{CNPq}$ & 8,40 & 2,07 \\
\hline & $\begin{array}{c}\text { Número de novas instituições } \\
\text { participantes por UF, por Edição do } \\
\text { PPSUS }\end{array}$ & $\begin{array}{l}\text { Número de instituições que } \\
\text { receberam recursos do PPSUS pela } \\
\text { primeira vez, por UF, por Edição do } \\
\text { PPSUS }\end{array}$ & $\mathrm{CNPq}$ & 8,80 & 1,79 \\
\hline & $\begin{array}{l}\text { Demanda bruta de projetos } \\
\text { submetidos, por UF, por Edição do } \\
\text { PPSUS }\end{array}$ & $\begin{array}{l}\text { Número de projetos submetidos, } \\
\text { por UF, por Edição do PPSUS }\end{array}$ & $\mathrm{CNPq}$ & 8,20 & 2,05 \\
\hline & $\begin{array}{l}\text { Número de projetos financiados, por } \\
\text { UF, por Edição do PPSUS }\end{array}$ & $\begin{array}{l}\text { Número de projetos financiados, } \\
\text { por UF, por Edição do PPSUS }\end{array}$ & $\mathrm{CNPq}$ & 8,20 & 2,05 \\
\hline & $\begin{array}{l}\text { Número de projetos financiados, a } \\
\text { cada R\$ } 100.000,00 \text { investidos, por } \\
\text { UF, por Edição do PPSUS }\end{array}$ & $\begin{array}{l}\text { Número de projetos financiados, } \\
\text { por UF, por Edição do PPSUS, a } \\
\text { cada R\$ } 100.000,00 \text { investidos }\end{array}$ & $\mathrm{CNPq}$ & 7,80 & 1,79 \\
\hline \multirow{2}{*}{$\begin{array}{l}\text { Resultado } \\
\text { (output) }\end{array}$} & $\begin{array}{l}\text { Verificação da média do número de } \\
\text { artigos científicos completos } \\
\text { publicados pelo pesquisador } \\
\text { contemplado nos cinco anos } \\
\text { anteriores e nos cinco posteriores ao } \\
\text { financiamento recebido pelo PPSUS, } \\
\text { por UF e por Edição do PPSUS. }\end{array}$ & $\begin{array}{l}\text { Média do número de artigos } \\
\text { científicos completos publicados } \\
\text { pelo pesquisador contemplado nos } \\
\text { cinco anos anteriores e nos cinco } \\
\text { posteriores ao financiamento } \\
\text { recebido pelo PPSUS, por UF e por } \\
\text { Edição do PPSUS e assim verificar } \\
\text { se houve algum impacto. }\end{array}$ & $\begin{array}{l}\text { Currículo } \\
\text { Lattes }\end{array}$ & 9,20 & 0,84 \\
\hline & $\begin{array}{l}\text { Verificação da média do número de } \\
\text { produção técnica do pesquisador } \\
\text { contemplado nos cinco anos } \\
\text { anteriores e nos cinco posteriores ao } \\
\text { financiamento recebido pelo PPSUS, } \\
\text { por UF e por Edição do PPSUS. }\end{array}$ & $\begin{array}{l}\text { Média do número de produção } \\
\text { técnica do pesquisador contemplado } \\
\text { nos cinco anos anteriores e nos } \\
\text { cinco posteriores ao financiamento } \\
\text { recebido pelo PPSUS, por UF e por } \\
\text { Edição e assim verificar se houve } \\
\text { algum impacto. }\end{array}$ & $\begin{array}{l}\text { Currículo } \\
\text { Lattes }\end{array}$ & 8,80 & 1,64 \\
\hline
\end{tabular}

Fonte: Elaborado pelas autoras (2021).

Tabela 2 - Matriz final com indicadores de insumo e resultado propostos para avaliação de demais programas de fomento descentralizado, operacionalizados via convênios. 


\begin{tabular}{|c|c|c|c|c|c|}
\hline $\begin{array}{c}\text { Tipo de } \\
\text { Indicador }\end{array}$ & Indicador & Descrição & $\begin{array}{c}\text { Fonte de } \\
\text { Informação }\end{array}$ & Média & $\begin{array}{l}\text { Desvio- } \\
\text { Padrão }\end{array}$ \\
\hline \multirow{7}{*}{$\begin{array}{l}\text { Insumo } \\
\text { (input) }\end{array}$} & $\begin{array}{l}\text { Total de recursos financeiros } \\
\text { federais por UF, por ano }\end{array}$ & $\begin{array}{c}\text { Valor de recursos federais } \\
\text { transferidos à UF, por meio do } \\
\text { programa avaliado, a cada ano }\end{array}$ & $\mathrm{CNPq}$ & 8,00 & 2,12 \\
\hline & $\begin{array}{l}\text { Total de recursos financeiros } \\
\text { estaduais por UF, por ano }\end{array}$ & $\begin{array}{l}\text { Valor de recursos estaduais } \\
\text { transferidos por UF, por ano }\end{array}$ & $\mathrm{CNPq}$ & 8,00 & 2,12 \\
\hline & $\begin{array}{c}\text { Número de instituições } \\
\text { participantes por UF, por } \\
\text { ano }\end{array}$ & $\begin{array}{l}\text { Número de instituições } \\
\text { participantes nos projetos } \\
\text { financiados, por UF, por meio do } \\
\text { programa avaliado, a cada ano }\end{array}$ & $\mathrm{CNPq}$ & 8,00 & 1,87 \\
\hline & $\begin{array}{c}\text { Número de novas } \\
\text { instituições participantes por } \\
\text { UF, por ano. }\end{array}$ & $\begin{array}{l}\text { Número de instituições que } \\
\text { receberam recursos do programa } \\
\text { avaliado pela primeira vez, por } \\
\text { UF, a cada ano }\end{array}$ & $\mathrm{CNPq}$ & 8,40 & 1,67 \\
\hline & $\begin{array}{l}\text { Demanda bruta de projetos } \\
\text { submetidos, por UF, por ano }\end{array}$ & $\begin{array}{l}\text { Número de projetos submetidos, } \\
\text { por UF, no programa avaliado, a } \\
\text { cada ano. }\end{array}$ & $\mathrm{CNPq}$ & 7,40 & 1,95 \\
\hline & $\begin{array}{l}\text { Número de projetos } \\
\text { financiados, por UF, por ano }\end{array}$ & $\begin{array}{c}\text { Número de projetos financiados, } \\
\text { por UF, por meio do programa } \\
\text { avaliado, a cada ano }\end{array}$ & $\mathrm{CNPq}$ & 7,40 & 1,95 \\
\hline & $\begin{array}{c}\text { Número de projetos } \\
\text { financiados, a cada } \mathrm{R} \$ \\
100.000,00 \text { investidos, por } \\
\text { UF, por ano }\end{array}$ & $\begin{array}{l}\text { Número de projetos financiados, } \\
\text { por UF, por meio do programa } \\
\text { avaliado, a cada R } \$ 100.000,00 \\
\text { investidos, por ano. }\end{array}$ & $\mathrm{CNPq}$ & 7,80 & 1,79 \\
\hline $\begin{array}{l}\text { Resultado } \\
\text { (output) }\end{array}$ & $\begin{array}{l}\text { Verificação da média do } \\
\text { número de artigos científicos } \\
\text { completos publicados pelo } \\
\text { pesquisador contemplado } \\
\text { nos cinco anos anteriores e } \\
\text { nos cinco posteriores ao } \\
\text { financiamento recebido no } \\
\text { programa avaliado, por ano. }\end{array}$ & $\begin{array}{l}\text { Média do número de artigos } \\
\text { científicos completos publicados } \\
\text { pelo pesquisador contemplado } \\
\text { nos cinco anos anteriores e nos } \\
\text { cinco posteriores ao } \\
\text { financiamento recebido por meio } \\
\text { do programa avaliado, por UF, e } \\
\text { assim verificar se houve algum } \\
\text { impacto. }\end{array}$ & $\begin{array}{l}\text { Currículo } \\
\text { Lattes }\end{array}$ & 8,80 & 1,64 \\
\hline
\end{tabular}

Fonte: Elaborado pelas autoras, 2021.

Em relação ao PPSUS, a Matriz final é composta por 07 indicadores de insumo (input) e 02 de resultado (output), conforme visualizado na Tabela 1. Este resultado diverge da Matriz de medidas avaliativas propostas por Oliveira (2008), que propôs 87 critérios/indicadores para avaliação do programa. Ressalta-se que o foco do presente trabalho é desenvolver indicadores para a gestão no âmbito do PPSUS, confirmando o entendimento de alguns autores de que o uso de indicadores auxilia a gestão das atividades de $\mathrm{C}, \mathrm{T} \& \mathrm{I}$ realizadas nas agências de fomento, assim como contribuem para melhorias contínuas dos processos organizacionais (OHAYON; ROSENBERG, 2014; BAHIA, 2021). Como nunca se estabeleceu uma série histórica acerca do PPSUS, pode-se iniciar, a princípio, com um menor número de indicadores e verificar a viabilidade. O número excessivo de indicadores é apontado por Bahia (2021) como um dos erros comuns para uso pela gestão das organizações, pois quanto menor o número de indicadores, mais fácil será para o gestor focar suas atividades.

A Matriz final sugerida para os demais programas também possui poucos componentes: 07 indicadores de insumo (input) e 01 de resultado (output), conforme demonstrado na Tabela 2. Os indicadores de insumo são os mesmos para o PPSUS. Não se pretende propor que a Matriz seja um instrumento rígido, uma vez que as áreas gestoras poderão estabelecer resultados específicos considerando os objetivos de cada programa. 
Em relação aos indicadores de resultado, ressalta-se a nota obtida para a média de artigos científicos publicados e o alto grau de consenso: 9,20 e 0,84 para o PPSUS e 8,80 e 1,64 para os outros programas, respectivamente. Este foi o indicador que obteve maior nota e maior grau de consenso.

No caso específico do PPSUS, foi incluído ainda o indicador referente à produção técnica e foram retirados os indicadores propostos referentes à apresentação em congressos e eventos científicos e resumos publicados em anais de congressos, pois receberam nota inferior a 7. A busca por novos meios de divulgação de informação científica é essencial para os gestores se apropriarem dos conhecimentos gerados pelas pesquisas financiadas. Becker, Rech e Reis (2018) verificaram que apenas dois, em cada cinco gestores da área da saúde, relatam utilizar as informações oriundas de artigos científicos e o principal fator alegado como barreira para a utilização é a falta de tempo. A produção técnica, por exemplo, por meio de cartilhas, relatórios, informes, em linguagem acessível, pode ser uma opção e deve ser incentivada pelos gestores do PPSUS. Trata-se, portanto, de um indicador pertinente para este programa.

A publicação de artigos científicos, contudo, é algo que norteia a ação dos pesquisadores, tendo em vista a ênfase que as agências de fomento atribuem às publicações como critérios de desempenho de pesquisadores e instituições de pesquisa (CAVALCANTE, 2009). Desse modo, é incontestável a permanência do indicador para qualquer ação executada no CNPq.

\section{Considerações finais}

O desenvolvimento desta pesquisa levou à elaboração de duas Matrizes contendo indicadores de insumo e resultado para o PPSUS e para os principais programas de fomento descentralizados, operacionalizados via Convênios no CNPq: PRONEM, PPP e PRONEX. A diversidade dos programas tornou difícil uma padronização de indicadores. $\mathrm{O}$ desenho das ações de fomento descentralizado em C\&T se apresenta com múltiplas características, com programas com diferentes objetivos específicos, com diferentes atores institucionais envolvendo diferentes legislações e, ainda, com diferentes modalidades de fontes de financiamento (CGEE, 2010).

Apesar disso, com o auxílio dos especialistas por meio da técnica de Conferência de Consenso, foi possível chegar a duas Matrizes contendo uma proposta de indicadores de insumo e resultado para uso pelo CNPq para os principais programas operacionalizados via convênios, até então inexistentes. Trata-se de um instrumento conciso, contendo as informações consideradas de alta importância para os gestores e que, até então, não existia para as ações de fomento descentralizado.

O processo de concepção de indicadores tem caráter político e deve estar condizente com as diretrizes, os interesses e as necessidades dos atores institucionais (ELIAS; SOUZA, 2006). Por isso, é possível apreender que o uso da técnica de Conferência de Consenso, com a participação de servidores do CNPq, mostrou-se apropriada, por oferecer a possibilidade de contemplar diferentes pontos de vista dos especialistas selecionados, além de propiciar a validação dos indicadores. Todavia, a utilização das técnicas de consenso, com uso de especialistas, não se constitui numa validação técnica, formal ou estatística daquilo que a informação realmente mede, mas é uma análise subjetiva daquilo que se pressupõe que seja medido com base nos próprios profissionais (UCHOA et al., 2008). 
Considerando que as Matrizes já foram validadas pelos especialistas por meio da técnica descrita neste trabalho, espera-se que seja realizado um estudo-piloto para se testar o uso das Matrizes propostas e assim verificar se os indicadores são viáveis e de fato atendem, na prática, às necessidades dos gestores do CNPq responsáveis pelos programas PPSUS, PPP, PRONEM e PRONEX.

\section{Referências}

BAHIA, L. O. Guia referencial para construção e análise de indicadores. Brasília: ENAP, 2021, 43 p.

BALBACHEVSKY, E. Descentralização e Integração do Fomento Público - Federalismo e Políticas de Ciência, Tecnologia e Inovação: especificidade setorial e marcos institucionais na experiência internacional. Brasília: Centro de Gestão e Estudos Estratégicos, 2008. 32 p.

BANZI, R. et al. Conceptual frameworks and empirical approaches used to assess the impact of health research: an overview of reviews. Health Research Policy and Systems, v. 9, n. 26, p. 1-10, jun. 2011. Disponível em: https://health-policysystems.biomedcentral.com/articles/10.1186/1478-4505-9-26. Acesso em: 15 mar. 2021.

BECKER, L. A.; RECH, C.R.; REIS, R. S. Acesso à informação para tomada de decisão com base em evidências segundo a percepção de secretários municipais de saúde do estado do Paraná, no ano de 2014. Cadernos de Saúde Pública, Rio de Janeiro, v. 34, n. 7, p 1-7, jul. 2018. Disponível em: https://www.scielo.br/scielo.php?script=sci_arttext\&pid=S0102311X2018000706001\&lng=pt\&tlng=pt. Acesso em: 15 mar. 2021.

BRASIL. Programa Pesquisa para o SUS: gestão compartilhada em saúde - Diretrizes Técnicas. 5. ed. Brasília: Ministério da Saúde, 2014. 64 p.

BRASIL. Portaria Interministerial $n^{\circ} 424$, de 30 de dezembro de 2016. Estabelece normas para execução do estabelecido no Decreto n ${ }^{\circ} 6.170$, de 25 de julho de 2007, que dispõe sobre as normas relativas às transferências de recursos da União mediante convênios e contratos de repasse, revoga a Portaria Interministerial no 507/MP/MF/CGU, de 24 de novembro de 2011 e dá outras providências. Diário Oficial da União: seção 1, Brasília, DF, ano 154, n. 1, p. 25 34, 02 jan. 2017.

BRASIL. Estratégia Nacional de Ciência, Tecnologia e Inovação 2016/2022: Sumário Executivo. Brasília: Centro de Gestão e Estudos Estratégicos, 2018. 40 p.

BRASIL. Portaria no 3020, de 19 de novembro de 2019. Altera a Portaria de Consolidação no 5/GM/MS, de 28 de setembro de 2017, para instituir o Programa Pesquisa para o SUS: Gestão Compartilhada em Saúde - PPSUS. Diário Oficial da União: seção 1, Brasília, DF, ano 157, n. 227, p. 51, 25 nov. 2019.

CAVAlCANTE, L. R. M. T.; AQUINO, M. C. Ciência e Tecnologia. Brasil em Números. Rio de Janeiro: IBGE/Centro de Documentação e Disseminação de Informações, v. 16, p. 315-323, 2008. 
CAVALCANTE, L. R. Políticas de ciência, tecnologia e inovação no Brasil: uma análise com base nos indicadores agregados. Rio de Janeiro: Secretaria de Assuntos Estratégicos da Presidência da República, 2009. Disponível em:

https://www.ipea.gov.br/portal/index.php?option=com_content\&view=article\&id=5001. Acesso em: 15 mar. 2021.

CENTRO DE GESTÃO E ESTUDOS ESTRATÉGICOS. Descentralização do fomento à ciência, tecnologia e inovação no Brasil. Brasília: Centro de Gestão e Estudos Estratégicos, 2010.154 p.

CENTRO DE GESTÃO E ESTUDOS ESTRATÉGICOS. Estudos de caso de programas financiados com recursos dos fundos setoriais de CT\&I: Pappe \& PPP. In: Descentralização do fomento público federal. Relatório final: 2008. Brasília: Centro de Gestão e Estudos Estratégicos, 2008. 276 p.

ELIAS, F. S.; SOUZA, L. Indicadores para monitoramento de pesquisa em saúde no Brasil. Ciência da Informação, Brasília, v. 35, n. 3, p. 218-226, set./dez. 2006. Disponível em: https://www.scielo.br/scielo.php?script=sci_arttext\&pid=S0100-

$19652006000300021 \& \operatorname{lng}=$ pt\&tlng=pt. Acesso em: 15 mar. 2021.

FRANCISCO, L. T. S. T. Indicadores para avaliação de resultados de projetos de pesquisa científica e tecnológica. 2002. Dissertação (Mestrado em Administração) - Escola de Administração, Universidade Federal do Rio Grande do Sul, Porto Alegre, 2002. Disponível em: https://lume.ufrgs.br/handle/10183/2236. Acesso em: 15 mar. 2021.

MELO, J. N.; SANTANA, J. R.; SILVA, G. F. Ciência, tecnologia e inovação no Brasil: uma análise inter-regional por meio de indicadores. Revista Brasileira de Gestão e Desenvolvimento Regional, Taubaté, v. 15, n. 1, p. 76-90, jan./abr. 2019. Disponível em: https://www.rbgdr.net/revista/index.php/rbgdr/article/view/4321. Acesso em: 15 mar. 2021.

NICKEL, D. A. et al. Disseminação e uso dos resultados de pesquisas financiadas pela Secretaria de Ciência, Tecnologia e Insumos Estratégicos do Ministério da Saúde, Brasil, 2004 a 2007. Saúde em Debate. Rio de Janeiro, v. 41, n. 112, p. 208-220, jan./mar. 2017. Disponível em: https://www.scielo.br/scielo.php?script=sci_arttext\&pid=S0103$11042017000100208 \& \operatorname{lng}=$ pt\&tlng=pt. Acesso em: 15 mar. 2021.

OHAYON, P.; ROSENBERG, G. Análise dos indicadores de ciência, tecnologia e inovação no âmbito da Fundação Oswaldo Cruz (Fiocruz). Revista do Serviço Público, Brasília, v. 65, n. 3, p. 297-319, jul./set. 2014. Disponível em:

https://revista.enap.gov.br/index.php/RSP/article/view/626. Acesso em: 15 mar. 2021.

OLIVEIRA, M. M. Programa pesquisa para o SUS: gestão compartilhada em Saúde PPSUS: construção do modelo lógico e da matriz de medidas avaliativas. 2008. Dissertação (Mestrado em Ciências da Saúde) - Faculdade de Ciências da Saúde, Universidade de Brasília, Brasília, 2008. Disponível em: https://repositorio.unb.br/handle/10482/5231. Acesso em: 15 mar. 2021. 
SILVA, A. C. Descentralização em política de ciência e tecnologia. Estudos Avançados, São Paulo, v. 14, n. 39, p. 61-73, maio/ago. 2000. Disponível em: https://www.scielo.br/scielo.php?script=sci_arttext\&pid=S0103$40142000000200007 \& \operatorname{lng}=$ pt\&tlng=pt. Acesso em: 15 mar. 2021.

SOUZA, L. E. P. F; CONTANDRIOPOULOS, A. P. O uso de pesquisas na formulação de políticas de saúde: obstáculos e estratégias. Cadernos de Saúde Pública, Rio de Janeiro, v. 20, n. 2, p. 546-554, mar./abr. 2004. Disponível em: https://www.scielo.br/scielo.php?script=sci_arttext\&pid=S0102311X2004000200023\&lng=pt\&tlng=pt. Acesso em: 15 mar. 2021.

SOUZA, L. E. P. F; VIEIRA-DA-SILVA, L. M; HARTZ, Z. M. A. Conferência de Consenso sobre a imagem-objetivo da descentralização da atenção à saúde no Brasil. In: HARTZ, Z. M. A; VIEIRA-DA-SILVA, L. M. (org.). Avaliação em saúde dos modelos teóricos à prática na avaliação de programas e sistemas de saúde. Salvador: EDUFBA, Rio de Janeiro: Fiocruz, 2005. p. 65-102.

TOBAR, F.; YALOUR, M. R. Como fazer teses em saúde pública: conselhos e ideias para formular projetos e redigir teses e informes de pesquisas. Rio de Janeiro: Fiocruz, 2001. 172 p.

UCHOA, A. C. et al. Utilizando técnicas de consenso: potencialidades e limites na avaliação de informações em saúde. In: HARTZ, Z. M. A.; FELISBERTO, E.; VIEIRA-DA-SILVA, L. M. (org.). Meta-Avaliação da Atenção Básica Teoria e Prática. Rio de Janeiro: Fiocruz, 2008. p. 253-282.

Recebido em abril de 2021.

Aprovado em outubro de 2021. 